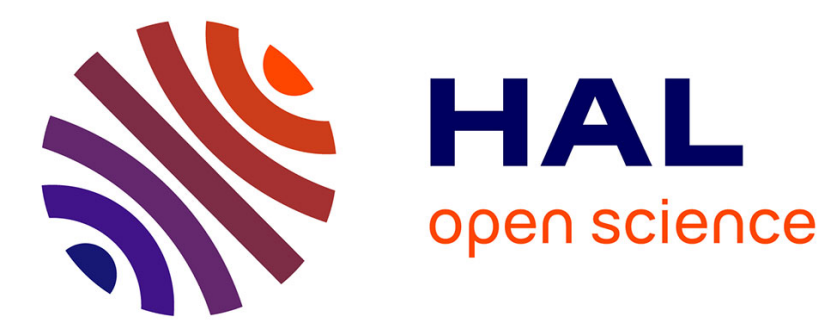

\title{
Production de chlore et de soude par le procédé à membrane échangeuse d'ions
}

François Delmas

\section{To cite this version:}

François Delmas. Production de chlore et de soude par le procédé à membrane échangeuse d'ions. Journal de Physique IV Proceedings, 1994, 04 (C1), pp.C1-223-C1-232. 10.1051/jp4:1994116 . jpa00252458

\section{HAL Id: jpa-00252458 https://hal.science/jpa-00252458}

Submitted on 1 Jan 1994

HAL is a multi-disciplinary open access archive for the deposit and dissemination of scientific research documents, whether they are published or not. The documents may come from teaching and research institutions in France or abroad, or from public or private research centers.
L'archive ouverte pluridisciplinaire HAL, est destinée au dépôt et à la diffusion de documents scientifiques de niveau recherche, publiés ou non, émanant des établissements d'enseignement et de recherche français ou étrangers, des laboratoires publics ou privés. 


\section{Production de chlore et de soude par le procédé à membrane échangeuse d'ions}

\section{F. DELMAS}

ELF ATOCHEM, Centre de Recherches Rhône-Alpes, BP. 63, 69493 Pierre-Bénite cedex, France

In Europe, $50 \%$ of the production of the chemical industry depends on chlorine. Elf Atochem, the french leader in chlorine, has chosen to extend its chlorine capacity with the ion exchange membrane (IEM) process.

The IEM have to be resistant to the aggressive electrolyte medium, have a very high permselectivity and conductivity. The chlor-alkali membranes are made of perfluorocarbon polymers with cationic exchange groups, sulfonic on the anodic side and carboxylic on the cathodic side. Their properties are well described by the cluster network model. The electrolyser and membrane evolution allow to obtain high caustic soda current efficiency ( $97 \%$ ) and a voltage of about $3 \mathrm{~V}$ at $3 \mathrm{kA} / \mathrm{m}^{2}, 90^{\circ} \mathrm{C}$ and with a caustic soda concentration of about $35 \mathrm{wt} \%$. Since 1990 , a 50 wt \% caustic soda solution can be produced directly in elecrolysers using a new generation of multilayer ion exchange membrane.

Despite the unfavorable economic climate in which the chlorine industry finds itself (overcapacity, environment), the IEM process has made a remarquable breakthrough : this technology appears to be the most economic and above all the most respectful of the environment. This process is already recognized as the substitute of the mercury process.

Elf Atochem operates two chlor-alkali membrane plants : one of $80 \mathrm{kt} \mathrm{Cl}_{2}$ /year in USA and a second of $120 \mathrm{kt} \mathrm{Cl}_{2} /$ year in France. 
L'industrie du chlore joue un rôle prépondérant dans le développement industriel et économique mondial. Aujourd'hui, plus de $50 \%$ du chiffre d'affaires, plus de $30 \%$ des investissements et environ $25 \%$ des emplois de l'industrie chimique mondiale reposent sur les activités chlore, soude et dérivés. [1].

Elf Atochem, premier chlorier français, quatrième au rang européen et sixième au rang mondial dispose d'une capacité annuelle de production de 1,2 Mt de chlore. La capacité mondiale est estimée à plus de $44 \mathrm{Mt}$ de chlore [2].

\section{Procédés d'électrolyse}

Nous exploitons les trois types de procédé permettant de produire du chlore et de la soude par électrolyse.

Le procédé à cathode de mercure consiste à produire du chlore et un amalgame $\mathrm{Hg}-\mathrm{Na}$ dans une cellule d'électrolyse, l'hydrogène et la soude dans un réacteur distinct : le décomposeur. Il présente l'inconvénient de mettre en oeuvre un produit toxique : le mercure. En revanche, il permet de produire directement de la soude à $50 \%$ quasiment exempte de chlorure.

Le procédé à diaphragme percolant consomme moins d'énergie électrique que le procédé à cathode de mercure. En revanche, il nécessite une consommation élevée de vapeur pour concentrer la soude au titre commercial de $50 \%$. De plus, celle-ci contient "in fine" environ $1 \%$ de sel.

La pollution de la soude par du chlorure (procédé diaphragme) et les problèmes d'environnement (mercure) ont incité les producteurs de chlore à explorer une troisième voie dans laquelle la cellule d'électrolyse est compartimentée à l'aide d'une membrane échangeuse d'ions.

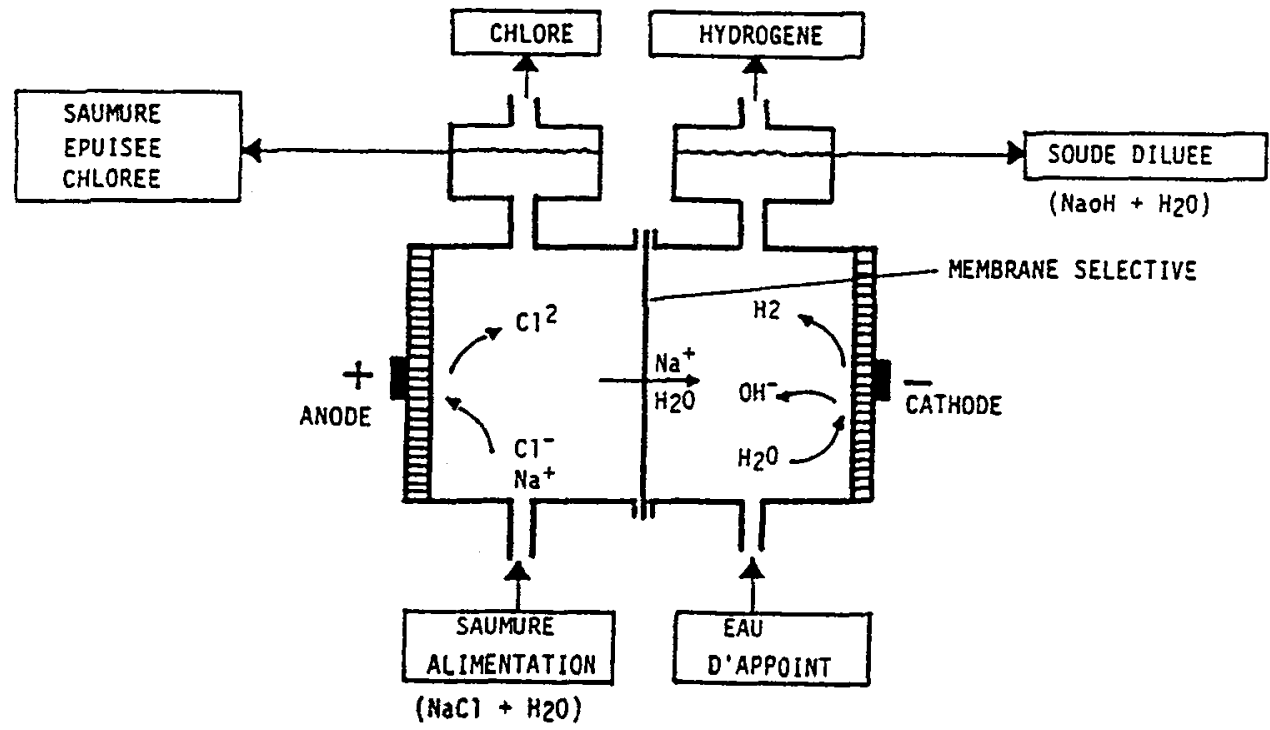

Schéma de principe de fonctionnement 
Le compartiment anodique de la cellule est alimenté par une saumure épurée et saturée en $\mathrm{NaCl}$ $(26 \%)$. Le chlorure est oxydé en chlore à l'anode et l'ion sodium solvaté migre sous l'effet du champ électrique du compartiment anodique vers le compartiment cathodique à travers une membrane échangeuse de cations. L'eau alimentant le compartiment cathodique est réduite à la cathode avec coproduction d'hydrogène et d'hydroxyde.

\section{TECHNOLOGIE}

Tous les électrolyseurs industriels sont constitués d'un empilement de $\mathrm{n}$ cellules élémentaires (anode + membrane + cathode) dans un électrolyseur de type filtre-presse. Il existe deux arrangements électriques. Lorsque les cellules élémentaires sont montées en série d'un point de vue électrique, l'électrolyseur est dit "bipolaire". Elf Atochem a donné sa préférence aux électrolyseurs monopolaires dans lesquels les cellules sont montées en parallèle.

L'amélioration des diverses parties de l'électrolyseur telles que les anodes dimensionnellement stables (DSA), les cathodes activées, les répartiteurs de courant, la maîtrise de la distance interélectrodes, de l'hydrodynamique... a permis le design de cellules d'électrolyse plus compactes et pouvant travailler sous de forte densité de courant $\left(3-5 \mathrm{kA} / \mathrm{m}^{2}\right)$

Bien que les électrolyseurs puissent être de tailles et de conceptions différentes [3-4-5] (tableau ci-dessous), leurs performances sont très voisines et dépendent principalement du type de membrane utilisée.

\begin{tabular}{|l|l|l|l|}
\hline \multicolumn{1}{|c|}{ Cellule } & $\begin{array}{l}\text { ML 60 } \\
\text { ASAFI } \\
\text { CHEMICAL }\end{array}$ & $\begin{array}{l}\text { FM 21 } \\
\text { I.C.I. }\end{array}$ & $\begin{array}{l}\text { DD 175 } \\
\text { O.D.N.T. }\end{array}$ \\
Configuration électrique & Bipolaire & Monopolaire & Monopolaire \\
Surface active & $5,4 \mathrm{~m}^{2}$ & $0,21 \mathrm{~m}^{2}$ & $1,75 \mathrm{~m}^{2}$ \\
Recirculation & forcée (pompe) & forcée (pompe) & naturelle(gaz lift) \\
\hline
\end{tabular}

\section{MEMBRANES ECHANGEUSES D'IONS}

Ces membranes doivent posséder un ensemble de qualité difficile à réunir en un seul matériau :

$\vartheta$ une bonne tenue mécanique,

$\supset$ une stabilité chimique vis-à-vis du chlore, de la soude et de l'hypochlorite à $90^{\circ} \mathrm{C}$,

$\vartheta$ un nombre de transport le plus proche possible de 1 pour l'ion sodium,

จ "a contrario", être une barrière quasi absolue pour les ions hydroxyde,

$\checkmark$ une conductivité électrique élevée,

$\vartheta$ une durée de vie la plus grande possible. 
C'est au début des années 60 que démarre réellement l'ère des membranes perfluorées homogènes échangeuses de cations avec les travaux de du Pont appliqués aux piles à combustible. La première membrane employée dans une cellule chlore soude était une Nafion perfluorosulfonique de du Pont en 1964 [6]. Les performances de cette membrane étaient médiocres.

On doit aux chercheurs de du Pont, la théorie dite "cluster network model" qui rend compte convenablement des propriétés de ces membranes. Ce modèle propose une séparation du solvant et des sites échangeurs de la matrice fluorocarbonée en structures micellaires inverses, interconnectées par des canaux courts et fins. Cette théorie est utilisée pour décrire les phénomènes de transport et la rejection des ions $\mathrm{OH}^{-}$.

Elle permet d'expliquer la contradiction apparente entre un rendement faraday élevé et une concentration membranaire élevée en ions $\mathrm{OH}^{-}$. La portée des interactions électrostatiques des groupes ioniques fixes est voisine de $5 \AA$ dans les conditions où se trouve placée la membrane. La théorie prévoit aussi que le diamètre des clusters, le nombre de groupes ioniques par Cluster et le nombre de molécules d'eau associées à chaque groupe varient comme la capacité d'échange de la membrane (Nafion). La barrière de potentiel que l'ion hydroxyde devra surmonter pour traverser la membrane sera donc d'autant plus élevée que le taux de gonflement de la membrane sera plus faible.

\section{Membranes asymétriques}

La première modification a consisté à réduire la capacité d'échange côté soude afin d'abaisser l'adsorption de l'eau et en définitif de diminuer la taille des clusters. Les performances étaient encore insuffisantes : rendement de $85 \%$ pour une soude à $20 \%$ en poids.

L'étape suivante a porté sur la modification chimique de la face cathodique de la membrane. Les groupes $-\mathrm{SO}_{3} \mathrm{H}$ sont transformés en sulfonamides $-\mathrm{SO}_{2} \mathrm{NHR}$ par action d'une amine primaire : le rendement atteint alors $90 \%$ pour une soude à $28 \%$ sans augmentation de la tension.

\section{Membranes carboxyliques}

Dès 1974, les sociétés japonaises Asahi Glass et Asahi Chemical présentaient une nouvelle membrane perfluorée comportant des groupes fonctionnels carboxyliques.

Cette membrane gonfle beaucoup moins que les polymères sulfoniques tout en atteignant des capacités d'échanges élevées ; avec ce type de membrane, l'arrêt des ions $\mathrm{OH}^{-}$est quasi parfait. Elle présente toutefois un inconvénient majeur : en milieu acide (anolyte), la dissociation des groupes fixes n'est pas complète, ce qui diminue la conductivité de la membrane et augmente la chute ohmique.

\section{Membranes bicouches}

Dès lors, une solution satisfaisante paraît être l'association d'une membrane sulfonique d'environ $200 \mu \mathrm{m}$ d'épaisseur, côté chlore et d'une membrane carboxylique côté soude. Cette dernière dont le rôle est celui de barrière absolue pour les ions hydroxyde, présente une plus faible conductivité électrique. Afin de conserver une conductivité acceptable, l'épaisseur de la membrane carboxylique doit être la plus mince possible, généralement comprise entre 10 et $50 \mu \mathrm{m}$. 


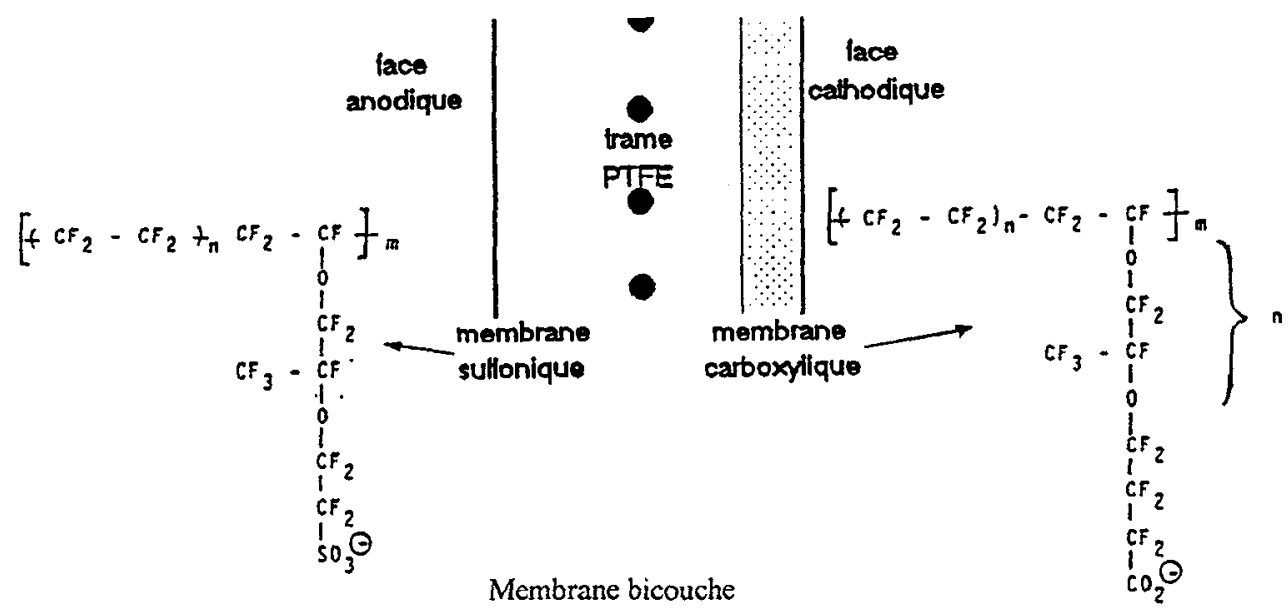

Ces membanes échangeuses d'ions bicouches permettent de produire, avec un bon rendement, de la soude à 32-35\%. En revanche, il n'est pas possible de générer au sein de l'électrolyseur de la soude à $50 \%$ en poids directement commercialisable [7].

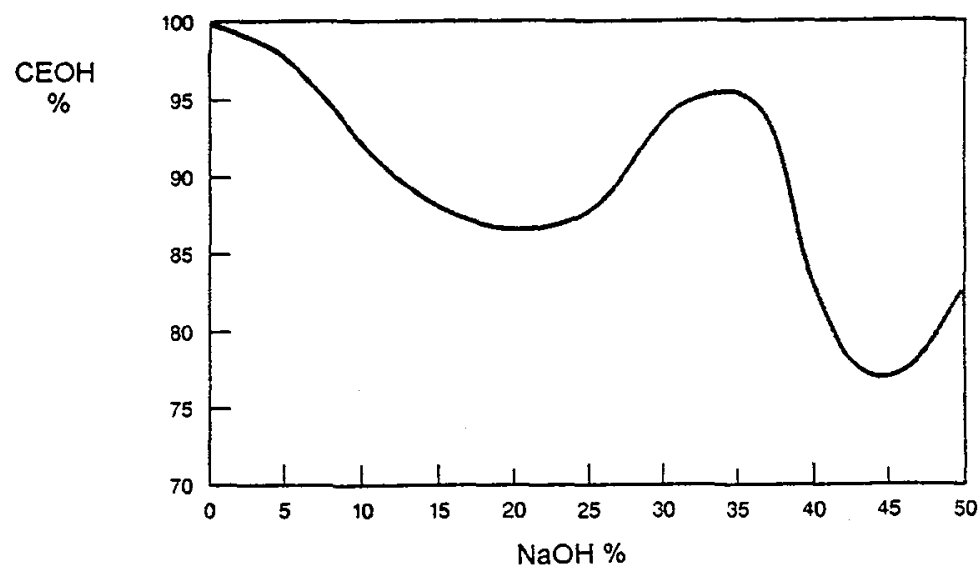

Rendement soude d'une membrane bicouche en fonction de la concentration en soude dans le catholyte.

Les performances de ces membranes sont très voisines, rendement de 96-97\% pour une soude à $32-35 \%$ en poids et une tension de $3.10 \mathrm{~V}$ (à $3 \mathrm{kA} / \mathrm{m}^{2}$ ). La durée de vie des membranes est de deux ans à une densité de courant de $3-4 \mathrm{kA} / \mathrm{m}^{2}$.

La membrane perfluorée étant hydrophobe, les gaz générés aux électrodes ont tendance à s'y accumuler. Afin d'éviter une augmentation de la chute ohmique par l'effet dit "de bulles" et une détérioration possible de la membrane (cloquage), la face anodique, et parfois cathodique, est hydrophylisée par l'incrustation en surface de particules inorganiques (oxydes).

L'hydrophilisation des deux faces d'une membrane permet de l'utiliser avec une technologie "gap zéro". C'est-à-dire que la distance entre la cathode et la membrane qui est plaquée contre l'anode, ne représente qu'une fraction de millimètre. 


\section{Performances}

En vingt ans, la consommation énergétique des cellules d'électrolyse à membrane a été divisée pratiquement par deux.

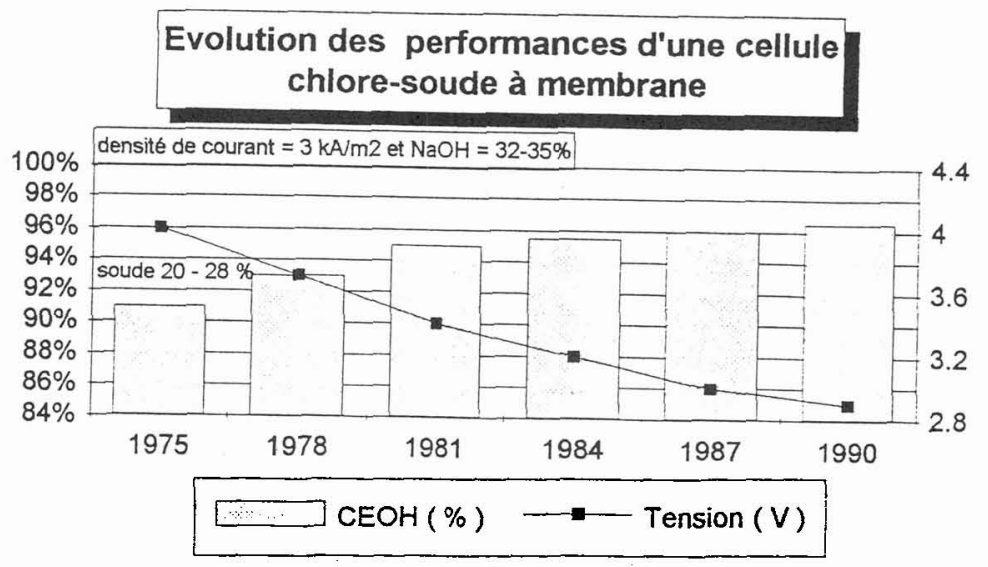

Dans les conditions de marche suivantes :

Anode DSA (Permelec),

$\checkmark$ Cathode activée,

จ Membrane échangeuse d'ions bicouche hydrophilisée en surface,

$\neg$ Gap zéro,

- Température $90^{\circ} \mathrm{C}$,

D Densité de courant $3 \mathrm{kA} / \mathrm{m} 2$,

$\rightarrow$ Soude $32-35 \%$,

la tension aux bornes de la cellule est alors d'environ 3.00-2.90 V, le rendement électrique de la production de soude est de $96-97 \%$ durant la première année. La consommation énergétique est alors d'environ $2050 \mathrm{kWh} / \mathrm{t}$ de $\mathrm{NaOH} 100 \%$. Rappelons que cette consommation était supérieure à $4000 \mathrm{kWh} / \mathrm{t}$ de $\mathrm{NaOH}$ au début des années 1970 . On ne note plus aujourd'hui de gain important au niveau énergétique.

Aujourd'hui, les fournisseurs de membranes pour les cellules d'électrolyse chlore-soude sont au nombre de trois : un américain, du Pont de Nemours et deux japonais, Asahi Chemical et Asahi Glass. Ils procèdent tous trois à un colaminage de deux membranes : l'une carboxylique, l'autre sulfonique. 


\section{Membranes échangeuses d'ions (soude à $50 \%$ )}

La dernière avancée technique dans le domaine des membranes "chlore-soude" a été réalisée par Asahi Glass[8-9]. Ce dernier a mis au point une nouvelle membrane qui permet de produire directement de la soude à $50 \%$. Cette membrane est constituée de trois couches : une face anodique classique à base sulfonique, une partie centrale comportant des groupes échangeurs carboxyliques et une face cathodique protégeant la membrane centrale de la soude à $50 \%$. Cette couche cathodique dite d'ajustement de la concentration est constituée du mélange d'un composé inorganique hydrophile et d'un polymère perfluoré sulfonique comme liant et échangeur cationique . La face cathodique rendue hydrophile, permet à la membrane de fonctionner en gap zéro

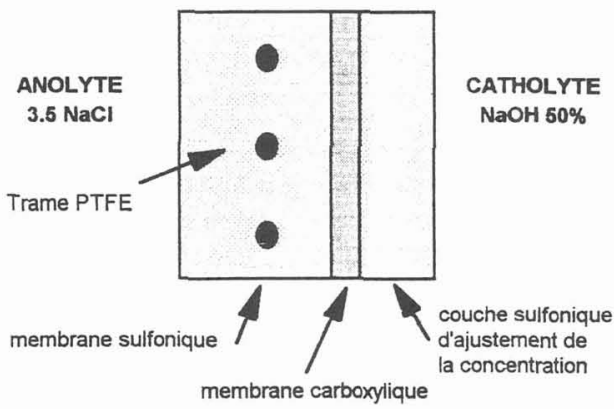

Membrane soude $50 \%$

\section{Performances électrochimiques}

Dans les conditions habituelles $\left(3 \mathrm{kA} / \mathrm{m}^{2}-90^{\circ} \mathrm{C}\right)$, la production directe de soude à $50 \%$ pénalise la tension de 300 à $400 \mathrm{mV}$ dont $120 \mathrm{mV}$ sont dus à une diminution du potentiel du dégagement d'hydrogène et à l'augmentation de la différence de potentiel de part et d'autre de la membrane [10]. Le rendement de la membrane reste élevé avec une valeur comprise entre 94 et $95 \%$.

$\mathrm{NaOH} 35 \%$

- tension théorique

- potentiel de membrane

- surtension anodique

- surtension cathodique

- chute ohmique (membrane)

- chute ohmique (électrolyte+gaz)
2,25
0,08
0,05
0,10
0,30
0,10

2,88
$\mathrm{NaOH} 50 \%$

$$
2,32
$$$$
0,12
$$$$
0,05
$$$$
0,10+\alpha
$$$$
0,30+\beta \quad \text { à }\{\mathrm{i}=3 \mathrm{kA} / \mathrm{m} 2
$$$$
0,10+\gamma
$$
$\{\mathrm{NaCl}=3,6 \mathrm{~N}$

$$
3,10-3,25
$$

Cette nouvelle membrane ouvre des perspectives intéressantes pour les utilisateurs . 


\section{COMPARAISON DES PROCEDES}

La technologie à membranes échangeuses d'ions présente divers avantages :

- Elle permet de produire une soude de bonne qualité quasiment exempte de chlorure (contrairement au procédé à diaphragme)

- Elle est plus respectueuse de l'environnement que le procédé à cathode de mercure.

\section{Qualité de la saumure}

La durée de vie d'une membrane échangeuse de cations, est intimement liée aux impuretés contenues dans la saumure et notamment, les éléments alcalino-terreux. Ainsi, il est impératif d'abaisser la teneur en calcium et en magnésium en-dessous de 20 ppb pour maintenir la permsélectivité et la bonne conductivité de la membrane. L'épuration "dite secondaire" sur résines échangeuses de cations permet d'atteindre correctement ce niveau de pureté. Le procédé à membrane nécessite une épuration beaucoup plus importante que les deux autres procédés qui acceptent une saumure primaire de qualité moindre .

\section{Consommation énergétique}

Chacun des procédés de production de chlore et de soude consomme de l'énergie électrique pour l'électrolyse et de la vapeur pour la concentration de la soude.

Il est clair que le procédé à membrane consomme moins d'énergie électrique que le procédé à diaphragme percolant ou à cathode de mercure. Il consomme aussi moins de vapeur que le procédé à diaphragme.

Globalement, le procédé à membrane consomme moins d'énergie que les deux autres procédés. Toutefois, la comparaison économique des différents procédés doit être considérée avec prudence, notamment le coût de la vapeur et de l'électricité sur le site de production doit être pris en compte.

\begin{tabular}{|c|c|c|c|c|c|}
\hline \multirow[t]{2}{*}{ Procédé } & \multirow{2}{*}{$\begin{array}{c}\text { Densité de } \\
\text { courant } \\
\left(\mathrm{kA} / \mathrm{m}^{2}\right)\end{array}$} & \multirow[t]{2}{*}{ Tension V } & \multicolumn{3}{|c|}{ Consommation d'énergie (1) } \\
\hline & & & Electrolyse & Concentration & Globale \\
\hline Mercure & 10 & 3,9 & 2700 & - & 2700 \\
\hline Diaphragme & 2,5 & 3,5 & 2500 & 600 & 3100 \\
\hline Membrane & 3 & 3 & 2100 & 150 & 2250 \\
\hline
\end{tabular}

(1) $\mathrm{kWh} / \mathrm{t} \mathrm{NaOH} 100 \%$ 


\section{CONCLUSION}

Le contexte géopolitique est déterminant et peut moduler fortement les considérations purement techniques citées précédemment. C'est la raison pour laquelle le passage à la technologie membrane est le plus souvent motivé par des critères non énergétiques, tels que l'absence de pollution de l'environnement (mercure), la qualité de la soude (faible teneur en sel) et l'approvisionnement du site en sel ou en saumure.

Maigré un contexte peu favorable de surcapacité mondiale en chlore [11], et de pression de l'environnement sur les dérivés chlorés, la technologie à membrane échangeuse d'ions dans le domaine de la production du chlore et de la soude fait une percée remarquable depuis la dernière décennie.

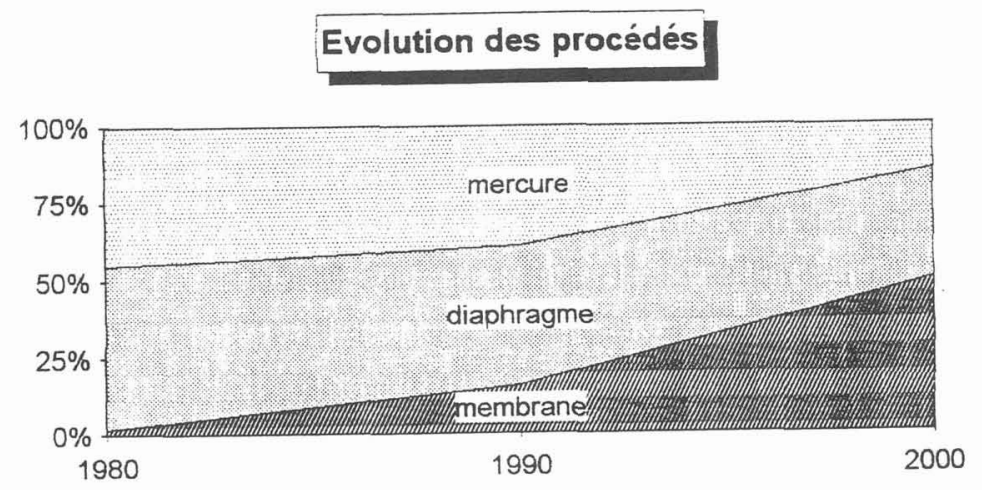

Il est à noter qu'au Japon, plus de $80 \%$ de la production de chlore est déjà assurée par le procédé à membrane. Cette nouvelle technologie s'impose d'ores et déjà comme le procédé de substitution au procédé à cathode de mercure. Elf Atochem dispose de deux ateliers chlore soude à membrane, le premier d'une capacité de 80000 t/an de $\mathrm{Cl}_{2}$ aux USA et le second d'une capacité de $120000 \mathrm{t} /$ an de $\mathrm{Cl}_{2}$ en France (Fos). 


\section{REFERENCES}

[1] (J. Puechal discours d'ouverture)

3ème Symposium mondial du chlore à Monte Carlo

[2] Savostianoff $D$, chlore/soude vers un déséquilibre durable ! Info chimie $\mathbf{n}^{\bullet} 310,1989$, p.207 - 233

[3] Asahi Chemical membrane chlor-alkali process (documentation Asahi Chemical)

[4] Cowell, G.W., Jackson C., Operating experience with the ICI FM 21 electrolyser, Modern chlor-alkali Techn. vol.3, p. 147

[5] Borrione P., Natural high flow rate recirculation, Modern Chlor-alkali Techn, vol.5 p.93104

[6] Grot W., Discovery and development of Nafion perfluorinated membranes..., Modern chlor-alkali techn, vol.3 p.123-130

[7] Yeager H.L., Gronowski A.A., Factors which influence the permselectivity of high performance chlor-alkali membranes, Modern chlor-alkali techn vol.5 p.81-92

[8] Sajima Y, Nakao M, Miyake H. Advances in Flemion membranes for chlor-alkali production

[9] Brevet EP 479-392 du 05.10.90

[10] Iishi T., Nakao M., Miyake H., Manufacture of $50 \%$ concentration caustic soda by ion exchange membrane technology, Kagaku Keizai, 1991, 38 (3), p.54-60

[11] Friedfeld B.A., The effect of green issues on the chlorine/caustic soda balance, Modern chlor-alkali techn.vol.5 p.1-12 